\title{
Modelo de Capacidade de Processo para o Software Público Brasileiro
}

\author{
Alessandra C. Zoucas ${ }^{1}$, Marcello Thiry ${ }^{1}$, Clenio F. Salviano ${ }^{2}$ \\ ${ }^{1}$ Universidade do Vale do Itajaí (UNIVALI) - Campus São José \\ Mestrado em Computação Aplicada (MCA) \\ Rodovia SC 407, km 04, São José - SC - Brasil \\ \{alessandrazoucas, marcello.thiry\}egmail.com
}

${ }^{2}$ Centro de Tecnologia da Informação Renato Archer (CTI)

Divisão de Melhoria de Processo e Qualidade de Software (DMPQS)

Rodovia D. Pedro I, km 143.6, Campinas - SP - Brasil

clenio.salvianoecti.gov.br

\begin{abstract}
This paper describes the development of a preliminary version of a Process Capability Model for the Brazilian Public Software. The model has been developed as a cooperative effort of universities, research groups and with the support of communities already subscribed at the Brazilian Public Software Portal. This work is coordinated by the Center for Information Technology Renato Archer in collaboration with the Software Quality and Productivity Laboratory/Univali. It had received funds from FINEP and it is part of a major project for the development of a Reference Model for Software Process Capability aligned to the characteristics and needs of the communities at the Brazilian Public Software Portal.
\end{abstract}

Resumo. Este artigo descreve o desenvolvimento da versão preliminar do Modelo de Capacidade de Processo para o Software Público Brasileiro. O modelo é construido cooperativamente e de forma compartilhada por universidades, grupos de pesquisa e com apoio das próprias comunidades do Portal do Software Público Brasileiro. Sua construção é coordenada pelo Centro de Tecnologia da Informação Renato Archer em parceria com Laboratório de Qualidade e Produtividade de Software da Univali. Este trabalho é financiado pela FINEP e integra o projeto de construção de um Modelo de Referência de Capacidade de Processo de Software alinhado às características e necessidades das comunidades do Portal do Software Público Brasileiro.

\section{Introdução}

Nos últimos anos a comunidade de software tem vivenciado a importância do processo para a qualidade do que é produzido. Segundo Fuggetta (2000) "a utilização de processos para tratar a inerente complexidade de software ganhou força a partir dos anos 1980" e ainda salientou que "a visão do desenvolvimento de software como um processo, tem ajudado significativamente a identificação das diferentes dimensões do desenvolvimento de software e os problemas que devem ser tratados para estabelecer práticas efetivas." Ainda de acordo com Fuggetta (2000), "nós temos de prestar atenção na complexa interpelação de numerosos fatores organizacionais, culturais, tecnológicos 
e econômicos [do desenvolvimento de software]". Neste sentido, a cada alteração significativa no âmbito do desenvolvimento de software, se faz necessário reavaliar as práticas atuais. Quais delas continuam válidas e significativas, quais precisam ser ajustadas ou se surgiram novas práticas a partir das ações bem sucedidas em um novo contexto.

O Software Público Brasileiro (SPB) é um conceito novo, onde o software é tratado como um bem público [Freitas 2009]. Sua produção é compartilhada e os resultados são disponibilizados à sociedade via Portal do Software Público Brasileiro ${ }^{1}$. Desta forma, o SPB vem induzindo uma mudança significativa no contexto da produção de software. O termo produção aqui deve ser considerado em um sentido mais amplo, incluindo não apenas o desenvolvimento de software, mas também a utilização, geração de conhecimento, distribuição de riquezas e demanda por soluções intensivas em software [Salviano et al. 2009a]. No final de 2008 foi aprovado o projeto de desenvolvimento do Modelo de Referência do Software Público Brasileiro. Este modelo tem como objetivo geral "aumentar a confiança em todos os níveis (legal, técnico, administrativo e de negócios) para os artefatos desenvolvidos, financiados ou geridos pelo governo, promovendo seu compartilhamento" [Júnior et al. 2009]. Ele é desenvolvido cooperativamente e de forma compartilhada por universidades, grupos de pesquisa e com apoio das comunidades do Portal do SPB.

Para a construção do Modelo de Referência do SPB foram definidos dez objetivos específicos a serem atingidos, onde cada um desses objetivos é tratado como um subprojeto. Um destes subprojetos visa desenvolver um modelo de capacidade de processo de software para o Software Público Brasileiro, contemplando um conjunto de orientações para o estabelecimento e utilização do modelo pela comunidade de interesse, até a fase de conclusão (entrega) de um produto [MCT 2007]. Este artigo apresenta a construção do modelo de capacidade de processo para o SPB que foi realizada em três fases:

- Fase 1. Construção de um Framework para apoiar a definição de métodos ${ }^{2}$ para a construção de Modelos de Capacidade de Processos e entendimento do SPB;

- Fase 2. Desenvolvimento de uma versão preliminar do Modelo de Capacidade de Processo para o SPB a partir do uso do framework supracitado;

- Fase 3. Revisão da versão inicial e desenvolvimento de uma nova versão do Modelo de Capacidade de Processo para o SPB.

A Fase 1 está completa e foi descrita em outro artigo [Salviano et al. 2009b]. As Fases 2 e 3 são descritas neste artigo. Na próxima seção são discutidas questões sobre Modelos de Capacidade de Processo. A Seção 3 apresenta o Software Público Brasileiro. A Seção 4 descreve brevemente o framework desenvolvido na Fase 1, bem como sua instanciação no método para a construção do modelo e os resultados da execução deste método. A Seção 5 apresenta a versão atual do modelo e a Seção 6 traz considerações finais do trabalho, apontando também os próximos passos.

\footnotetext{
${ }^{1}$ www.softwarepublico.gov.br

2 Procedimento regular, explícito e passível de ser repetido para conseguir-se alguma coisa, seja material ou conceitual [Bunge 1974].
} 


\section{Modelos de Capacidade de Processo}

Os Modelos de Capacidade de Processo existentes ou os mais conhecidos não atendem à diversidade de contextos e objetivos estratégicos das organizações [Salviano 2006], inclusive na área de software. Isto pode ser observado a partir da execução dos diversos projetos de construção e adaptações de modelos como, por exemplo: o MARES-MPE [Anacleto et al. 2004] que é uma adaptação da norma ISO/IEC 15504 [ISO/IEC 2003] para micro e pequenas empresas de software; o MARES-MINI/EI [Pickler et al. 2005] que é uma adaptação da norma ISO/IEC 15504 para micro empresas de software incubadas; o PRM.CBD [Tsukumo et al. 2006] que é a especialização de algumas áreas de processo dos modelos do CMMI [SEI 2006] e inclui processos do grupo de reuso do modelo da norma ISO/IEC 15504 [ISO/IEC 2003] para o desenvolvimento de software baseado em componentes; o Modelo para melhoria e avaliação da pesquisa em laboratórios universitários [Silva et al. 2007] para melhoria e avaliação da gestão estratégica de laboratórios universitários; o MR-MPS [SOFTEX 2009]; o Reference Model for the Leadership of Integrated Virtual Teams [Tuffley 2008] para estabelecer um modelo de capacidade de liderança de equipes virtuais integradas; o Automotive SPICE [AUTOMOTIVE SIG 2008] que é uma adaptação da norma ISO/IEC 15504, para estabelecer uma referência para avaliar a capacidade do processo de software de fornecedores de software automotivo; o COBIT Control Objectives for Information and related Technology [ITGI 2005] que é um framework constituído de práticas elaboradas a partir de consenso de especialistas no domínio de governança em TI e fornece medidas e modelos de maturidade para avaliar a execução das práticas. Mesmo os modelos de referência consolidados, depois de publicados, passam por evoluções e revisões periódicas. Podemos citar, por exemplo, o CMMI-DEV [SEI 2006] que está na sua terceira versão, o modelo CMM [Paulk et al. 1994], predecessor do CMMI-DEV, que teve 3 versões, e o modelo MR-MPS criado em 2005 atualmente na sua quarta revisão.

Além dos trabalhos de elaboração, adaptação e revisão de modelos existem demandas atuais de novos modelos de referência de processo. Como exemplos concretos, pode-se citar as iniciativas: Enterprise SPICE $^{3}$ que integrará normas existentes em um único modelo de referência de processo e modelo de avaliação de processo, voltados para processos empresariais; o Banking $\operatorname{SPICE}^{4}$ que integrará ferramentas metodológicas existentes e disponibilizará um mecanismo para a avaliação e melhoria dos processos implantados no setor bancário e financeiro internacional; o Modelo de Referência de Processo para a tele medicina [Hauck et al. 2008] que é um projeto de construção de um modelo de referência de processos de desenvolvimento de software para a tele medicina. Outra demanda atual é o Modelo de Capacidade de Processo para o Software Público Brasileiro [MCT 2007] que é descrito neste artigo.

\section{O Software Público Brasileiro - SPB}

O conceito de Software Público no Brasil tem seus primeiros registros de discussão na década de 1990 [Meffe 2009]. Atualmente, SPB é um dos fundamentos para definir a política de desenvolvimento e uso de software pelo setor público no Brasil [Meffe

\footnotetext{
${ }^{3} \mathrm{http}: / / \mathrm{www}$. enterprisespice.com/

${ }^{4} \mathrm{http}: / /$ www.bankingspice.com/
} 
2009]. Este conceito abrange a relação entre os órgãos públicos, em todos os estados brasileiros e demais esferas de poder, e destes com as empresas e a sociedade.

O conceito de software público diferencia-se do conceito de software livre em determinados aspectos, principalmente no que diz respeito à atribuição de bem público ao software [Alves et al. 2009]. Isto significa que há algum grau de responsabilidade por parte do governo brasileiro em garantir aos usuários do software condições apropriadas de uso do mesmo. Isto vem sendo realizado por meio da disponibilização de um conjunto de serviços básicos, via Portal do Software Público Brasileiro que oferece serviços como: página para cada comunidade; ferramenta de controle de versão; fóruns; lista de discussão para desenvolvimento e suporte; e outros. Além disto, também existem responsabilidades que são assumidas pela entidade que disponibiliza o software no portal como: prover um software com documentação de instalação e preparado para funcionar; disponibilizar um ponto focal ou uma equipe que possa fazer interlocução com a sociedade e receber suas demandas; construção de um ambiente virtual que operacionalize a comunicação com o usuário; gerir a colaboração que vai além da gestão da comunidade virtual associada ao software liberado; e realizar ações para incentivar a colaboração e gestão do conhecimento produzido.

Inicialmente, a intenção da iniciativa do SPB era acelerar a cooperação no governo, para reduzir os esforços de desenvolvimento, reduzir custos e racionalizar recursos. A tendência para a total liberação de soluções para a sociedade é recente. Atualmente, a iniciativa do SPB facilita a implantação de novas ferramentas nos diversos setores da administração pública, promove a integração entre as unidades federativas e oferece um conjunto de serviços públicos para sociedade com base no bem software [Meffe 2009].

\subsection{O Portal do SPB}

O Portal do Software Público Brasileiro disponibiliza diferentes soluções para áreas distintas, como por exemplo, a área da saúde, educação e saneamento, TV Digital e gestão de Tecnologia da Informação, entre outras. Estas soluções foram desenvolvidas por órgãos públicos, empresas ou universidades. O código fonte destas soluções é livre e seu acesso pode ser feito a partir do cadastro do interessado no portal do SPB. Este portal também hospeda Comunidades Virtuais que neste ambiente são denominadas de Comunidades de Aprendizagem, Trabalho e Inovação em Rede (CATIR). Uma CATIR é conceituada como grupos de indivíduos motivados por algum interesse ou propósito comum que se relacionam de forma colaborativa, continuada e em rede, presencialmente e/ou virtualmente, independentemente da localização física, visando compartilhar conhecimentos, aprender e gerar inovações no trabalho. Para obter o acesso às comunidades virtuais do ambiente CATIR também é necessário fazer o cadastramento no Portal e desta forma o indivíduo poderá escolher participar de comunidades existentes ou criar comunidades para apoio à sua organização. O Portal do SPB é dividido em três espaços: espaço do usuário que apresenta as funcionalidades voltadas para os usuários das soluções disponíveis no portal (alteração de configurações pessoais do usuário, lista de comunidades para o usuário poder se associar como membro, etc.); espaço comunidade que apresenta as funcionalidades pertinentes as comunidades (Fórum, Chat, Wiki, etc.) e o espaço do desenvolvedor que foi criado para que usuários com habilidade de desenvolvimento de código pudessem contribuir para o desenvolvimento de soluções disponíveis no portal SPB. 
Atualmente, no Portal do SPB estão cadastradas, como prestadoras de serviço, 88 empresas e 124 pessoas físicas autônomas. Qualquer prestador de serviço pode se cadastrar e associar um tipo de serviço a ser prestado. Ainda pode escolher a solução para a qual irá prestar o serviço que pode ser Instalação e Implantação, Consultoria, Treinamento, Desenvolvimento, Manutenção, Modelagem ou Suporte técnico. Em 12 de abril de 2009 o Portal SPB fez 2 anos de existência contando com mais de 40 mil pessoas com cadastro válido e 22 soluções de universidades públicas e privadas, de empresas, de prefeituras e da Câmara dos Deputados [Meffe 2009].

\subsection{Motivação para um modelo de capacidade de processo específico para o SPB}

Segundo Humphrey (1995), a qualidade do processo utilizado para construir um produto tem impacto na qualidade do que é produzido. Para apoiar uma avaliação da qualidade de processos, referências (benchmarking) são definidas visando propiciar uma objetiva e efetiva comparação dos processos avaliados. Estas referências podem ser estabelecidas a partir de modelos de capacidade de processo como os do CMMI (CMMI-DEV) [SEI 2006], da ISO/IEC 15504 [ISO/IEC 2003], da iniciativa de brasileira MPS.BR [SOFTEX 2009], entre outros.

A motivação para um modelo de capacidade de processo para o SPB surgiu para atender a demanda do governo federal em garantir que o software disponibilizado e evoluído pelas comunidades do portal SPB tenha qualidade. Entretanto, percebeu-se que nem todas as práticas ou resultados esperados existentes nos modelos de capacidade de processo disponíveis atualmente são aplicáveis no contexto de desenvolvimento do SPB. Construir um modelo de capacidade de processo específico para o SPB não significa somente adaptar os modelos de desenvolvimento e de serviços já existentes, mas desenvolver um modelo com as comunidades e a partir de experiências destas comunidades (de baixo para cima, da prática para a sistematização). Para isso, foram utilizadas também práticas de outros modelos existentes e de outras comunidades, com a tecnologia e experiência do CTI e de outros parceiros em desenvolvimento de modelos para outros domínios [Salviano et al. 2009a].

Desta forma, foram identificadas e definidas áreas de capacidade de processo específicas para o SPB como, por exemplo, Gestão da Comunidade (GDC) onde são formalizadas práticas para apoiar o funcionamento da Comunidade do portal SPB visando atingir os objetivos da Comunidade e do SPB. Práticas ou resultados esperados de modelos consolidados também foram identificados e adaptados para o contexto do SPB como, por exemplo, Solicitação de Melhoria (SDM) que é uma adaptação da área de processo Gerência de Requisitos disponível em outros modelos.

\section{Método para construir o Modelo de Capacidade de Processo para o SPB}

Esta seção apresenta o framework desenvolvido para a definição de métodos de construção de modelos de capacidade de processo, sua instanciação para definir o método de construção do modelo SPB, bem como as atividades executadas para a construção deste modelo. 


\subsection{PRO2PI-MFMOD}

A metodologia PRO2PI ${ }^{5}$ [Salviano 2006] foi acrescida de um novo elemento chamado Framework de Métodos para o Desenvolvimento de Modelos de Capacidade de Processo (PRO2PI-MFMOD) [Salviano et al. 2009b]. O PRO2PI-MFMOD ${ }^{6}$ tem sido desenvolvido no Laboratório de Qualidade e Produtividade de Software (LQPS) da Univali em parceria com o Centro de Tecnologia da Informação Renato Archer (CTI).

O PRO2PI-MFMOD foi gerado a partir dos relatos de criação de normas e modelos de capacidade de processo encontrados na literatura e que detalham seus métodos de construção [Salviano et al. 2009b]. Estes relatos foram estudados e compilados. A partir disto foram identificadas práticas e técnicas que são aplicadas para a construção de modelos de capacidade de processo que estão detalhados e disponíveis no PRO2PI-MFMOD [Salviano et al. 2009b] e [Zoucas et al. 2009]. Desta forma o PRO2PI-MFMOD disponibiliza um conjunto de quatro componentes independentes que têm uma relação pré-definida, com o propósito de elaborar métodos para a construção de modelos de capacidade de processo.

A partir da aplicação do framework, métodos poderão ser instanciados e adotados como procedimento regular, explícito e passível de ser repetido para construir modelos de capacidade de processos para domínios diferentes. A seguir são apresentados os quatro componentes PRO2PI-MFMOD:

1) Exemplos de Utilização - A versão atual do PRO2PI-MFMOD define dez Exemplos de Utilização (E1 a E10) apresentados como o mapeamento das atividades de dez métodos de construção de modelos de capacidade de processo a partir do PRO2PIMFMOD. O detalhamento de Exemplos de Utilização podem ser vistos em outro artigo [Salviano et al. 2009b].

2) Práticas Sequenciais - O PRO2PI-MFMOD define sete Práticas Sequenciais (P1 a P7) para orientar a definição de um método ou processo de construção de modelos de capacidade de processo:

- P1 Decisões iniciais: As decisões iniciais podem estar relacionadas com qualquer uma das seis práticas apresentadas a seguir. Tem como objetivo estabelecer um planejamento preliminar para a construção do novo modelo;

- P2 Análise de fontes: Aqui são identificadas, coletadas e analisadas fontes de informação sobre o contexto e as características de um segmento ou domínio específico para o qual o modelo será criado. Assim, se espera levantar, estudar e adquirir conhecimento a respeito de práticas deste segmento ou domínio;

- P3 Estratégia de desenvolvimento: Está relacionada com a definição da estratégia a ser utilizada para desenvolver o modelo. Uma questão fundamental é como a comunidade de interesse será envolvida nesse desenvolvimento.

- P4 Projeto do modelo: Diz respeito a projetar o modelo de capacidade de processo antes de ele ser construído e definir sua estrutura;

- P5 Desenvolvimento da versão preliminar do modelo: Visa desenvolver a versão preliminar do modelo. É o primeiro passo para se ter uma versão do modelo que possa ser efetivamente aplicada em projetos piloto;

\footnotetext{
${ }^{5}$ Process Capability Profile to drive Process Improvement

${ }^{6}$ Method Framework for Engineering Process Capability Models
} 
- P6 Validação da versão preliminar do modelo: A versão preliminar do modelo é aplicada em projetos piloto com o objetivo de identificar características e problemas que possam ser melhorados antes de lançar uma versão consolidada do modelo;

- P7 Consolidação do modelo: Depois que os problemas detectados na versão preliminar são corrigidos, a versão consolidada do modelo é desenvolvida. Esta versão consolidada pode ser novamente testada em novos projetos piloto.

3) Regras de Customização - O PRO2PI-MFMOD define sete Regras de Customização (RC1 a RC7) para orientar o ajuste das Práticas Sequenciais na instanciação de um método de construção de modelos de capacidade de processo:

(RC1) Uma atividade corresponde a uma prática (uma atividade para uma prática); (RC2) Não existe atividade correspondente à prática (nenhuma atividade para uma prática); (RC3) Não existem atividades que correspondam a uma ou mais práticas finais consecutivas, por que o ciclo de vida do método ou processo termina antes das práticas finais (nenhuma atividade para muitas práticas finais); (RC4) Duas ou mais atividades correspondem a uma prática (várias atividades para uma prática); (RC5) Uma atividade corresponde a duas ou mais práticas consecutivas, por que a atividade é mais geral e simplificada do que a customização da prática (uma única atividade para muitas práticas); (RC6) Existem atividades consecutivas que correspondem a ciclos de práticas consecutivas (ciclos de atividade em muitas práticas); e (RC7) Existe uma ou mais técnicas especificada para uma ou mais atividades.

4) Técnicas Específicas - A versão atual do PRO2PI-MFMOD define doze exemplos de Técnicas Específicas (T1 a T12) que podem ser adotadas para apoiar a construção de modelos de capacidade de processos: (T1) Tradução de áreas de processo; (T2) Questionário; (T3) Revisão da literatura; (T4) Análise de trabalhos correlatos; (T5) Estudo de caso; (T6) Representação de domínio; (T7) Abstração de processos; (T8) Tradução de modelos no formato de requisitos; (T9) Brainstorming (tempestade de ideias); (T10) Revisão pelos pares; (T11) Entrevista; (T12) Workshop (oficina). O detalhamento das técnicas pode ser visto em outro artigo [Zoucas et al. 2009].

\subsection{Instanciação do Framework de Métodos}

A partir da instanciação do PRO2PI-MFMOD [Salviano et al. 2009b] foi gerado um método para a construção do modelo de capacidade de processos específico para o SPB. Foram usados os 4 componentes do PRO2PI-MFMOD.

Os Exemplos de Utilização disponíveis no PRO2PI-MFMOD foram úteis para observar experiências anteriores de diferentes métodos. Foi possível identificar como as atividades dos métodos estudados estão distribuídas dentre as Práticas Sequenciais. Também, permitiu avaliar as Técnicas Específicas mais empregadas por prática. Observando tais exemplos, e o contexto em que cada um deles havia sido aplicado, algumas decisões para apoiar a instanciação do método foram tomadas. Foi estabelecido que o método fosse constituído de ciclos de práticas consecutivas visando refinar o resultado obtido a cada ciclo. Esta decisão foi tomada, por exemplo, para garantir que domínio seria compreendido pela equipe, uma vez que a equipe não conhecia o domínio do SPB. Desta forma, foram estabelecidos três ciclos para a prática (P2) Análise de fontes. Isto caracteriza que o método adota a Regra de Customização 6 (RC6): ciclos de atividade em muitas práticas. Também foi determinado que cada atividade do método 
correspondesse a uma única Prática Sequencial e assim garantir que o método tenha atividades objetivas. Desta forma, o método adota a Regra de Customização 1 (RC1) onde uma atividade corresponde a uma prática.

O método definido para a construção do modelo de capacidade de processo para o SPB é constituído de quatorze atividades: (1) decisões iniciais; (2) identificação de fontes e análises iniciais; (3) estratégia de desenvolvimento; (4) análises detalhadas das fontes identificadas; (5) detalhamento da estratégia; (6) projeto do modelo em alto nível; (7) revisão das fontes e novas análises; (8) revisão da estratégia; (9) projeto do modelo; (10) desenvolvimento da versão preliminar do modelo; (11) validação inicial; (12) desenvolvimento da versão preliminar do modelo; (13) validação; (14) consolidação do modelo. A figura 1 apresenta as atividades deste método em relação às Práticas Sequenciais do PRO2PI-MFMOD.

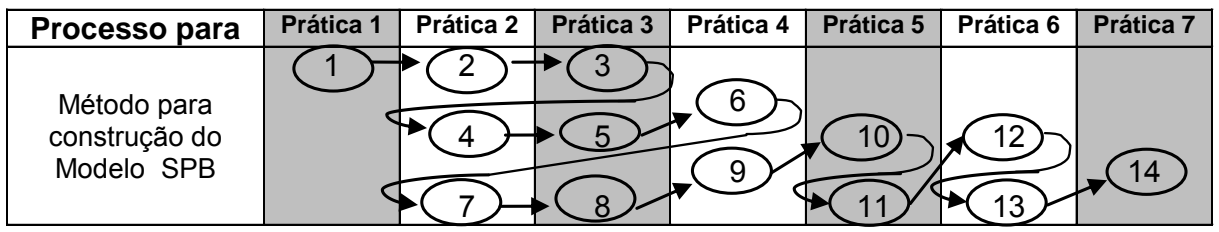

\section{Figura 1. Praticas do PRO2PI-MFMOD e atividades de um processo para o Modelo de Referência de Capacidade de Processo para o SPB}

O PRO2PI-MFMOD também apoiou a seleção das Técnicas Específicas que foram utilizadas na execução deste método. Foram selecionadas as Técnicas Específicas mais empregadas por prática dos métodos estudados ou a Técnica Específica considerada mais apropriada para apoiar uma determinada Prática Sequencial. Entre as doze Técnicas Específicas disponíveis no PRO2PI-MFMOD, seis foram selecionadas: (T3) Revisão da literatura; (T9) Brainstorming; (T7) Abstração de processo; (T10) Revisão pelos pares e (T12) Workshop. Desta forma, o método definido adota a Regra de Customização 1 (RC7) disponibilizada como um componente do PRO2PI-MFMOD.

A tabela 1 apresenta a relação entre as Práticas Sequenciais do PRO2PIMFMOD e as Técnicas Específicas aplicadas durante a execução do método.

Tabela 1: Práticas do PRO2PI-MFMOD e técnicas do método para o Modelo SPB

\begin{tabular}{|c|c|c|c|c|c|c|c|}
\hline Práticas & $\begin{array}{c}\text { P1: Decisões } \\
\text { iniciais }\end{array}$ & $\begin{array}{c}\text { P2: } \\
\text { Análise de } \\
\text { fontes }\end{array}$ & $\begin{array}{c}\text { P3: } \\
\text { Estratégia de } \\
\text { desenvolvi } \\
\text { mento }\end{array}$ & $\begin{array}{c}\text { P4: } \\
\text { Projeto do } \\
\text { modelo }\end{array}$ & $\begin{array}{c}\text { P5: } \\
\text { Desenvolvim } \\
\text { ento da } \\
\text { versão } \\
\text { preliminar } \\
\text { do modelo }\end{array}$ & $\begin{array}{c}\text { P6: } \\
\text { Validação da } \\
\text { versão } \\
\text { preliminar } \\
\text { do modelo }\end{array}$ & $\begin{array}{c}\text { P7: } \\
\text { Consolida } \\
\text { ção do } \\
\text { modelo }\end{array}$ \\
\hline $\begin{array}{c}\text { Técnicas } \\
\text { Modelo SPB }\end{array}$ & - & $\begin{array}{c}\text { T3: } \\
\text { Revisão da } \\
\text { Literatura }\end{array}$ & $\begin{array}{c}\text { T9: } \\
\text { Brainstorming }\end{array}$ & $\begin{array}{c}\text { T7: Abstração } \\
\text { de processo e } \\
\text { T9: } \\
\text { Brainstorming }\end{array}$ & $\begin{array}{c}\text { T7: Abstração } \\
\text { de processo } \\
\text { eT10: Revisão } \\
\text { pelos pares }\end{array}$ & $\begin{array}{c}\text { T12: } \\
\text { Workshop e } \\
\text { T5: } \\
\text { Estudo de } \\
\text { Caso }\end{array}$ & $\begin{array}{c}\text { Estudo de } \\
\text { Caso }\end{array}$ \\
\hline
\end{tabular}

Durante a análise dos métodos estudados, práticas comumente executadas foram levantadas e se tornaram as Práticas Sequenciais disponíveis no PRO2PI-MFMOD. Estas Práticas Sequenciais auxiliaram na identificação de sete critérios utilizados para avaliar os métodos estudados. Estes critérios também foram aplicados na avaliação do método definido para a construção do modelo SPB. Como resultados desta avaliação o método demonstrou atender totalmente a cada um dos critérios. A tabela 2 resume os resultados da avaliação. 
Tabela 2: Avaliação do método

\begin{tabular}{|l|l|l|}
\hline Critério & Atividades & Avaliação \\
\hline $\begin{array}{l}\text { 1. Existe documentação sobre } \\
\text { como as decisões iniciais foram } \\
\text { realizadas. }\end{array}$ & 1 & $\begin{array}{l}\text { O método prevê que as decisões iniciais sejam realizadas na execução de } \\
\text { um passo. }\end{array}$ \\
\hline 2. Houve estudo da literatura & 2,4 e 7 & $\begin{array}{l}\text { O método prevê que o estudo da literatura seja realizado em três passos, } \\
\text { onde as fontes são identificadas e analisadas, em seguida são realizadas } \\
\text { análises detalhadas das fontes identificadas e por fim é feito uma revisão } \\
\text { das fontes e novas análises são conduzidas. }\end{array}$ \\
\hline $\begin{array}{l}\text { 3. Existe uma estratégia para o } \\
\text { desenvolvimento do modelo }\end{array}$ & 3,5 e 8 & $\begin{array}{l}\text { O método prevê que uma estratégia para o desenvolvimento do modelo seja } \\
\text { definida em três passos, onde uma estratégia de desenvolvimento alto nível } \\
\text { é traçada, em seguida é feito o detalhamento desta estratégia e por fim ela é } \\
\text { revisada. }\end{array}$ \\
\hline $\begin{array}{l}\text { 4. Houve definição da estrutura do } \\
\text { modelo }\end{array}$ & 6 e 9 & $\begin{array}{l}\text { O método prevê a definição da estrutura do modelo que será construído em } \\
\text { dois passos, onde é realizado o projeto do modelo em alto nível e em } \\
\text { seguida é realizada uma revisão e detalhamento do projeto do modelo. }\end{array}$ \\
\hline $\begin{array}{l}\text { 5. Foi desenvolvida uma versão } \\
\text { preliminar }\end{array}$ & 10 e 11 & $\begin{array}{l}\text { O método prevê o desenvolvimento de uma versão preliminar do modelo e } \\
\text { uma avaliação inicial do mesmo. }\end{array}$ \\
\hline 6. O modelo foi validado & 12 e 13 & $\begin{array}{l}\text { O método prevê a validação do modelo preliminar do modelo em dois } \\
\text { passos, onde será realizado a aplicação da versão preliminar do modelo e } \\
\text { em seguida a sua validação. }\end{array}$ \\
\hline 7. O modelo está consolidado & 14 & $\begin{array}{l}\text { O método prevê a consolidação do modelo em um passo, onde será } \\
\text { realizada aplicação e a consolidação do modelo. }\end{array}$ \\
\hline
\end{tabular}

\subsection{Execução do Método para o Modelo de Capacidade de Processo do SPB}

Como resultado da execução do método definido, foi possível construir e avaliar uma versão preliminar do modelo para o SPB. Esta seção apresenta como o método foi executado a partir das sete Práticas Sequenciais definidas pelo PRO2PI-MFMOD.

P1 - Decisões Iniciais: assim que este subprojeto foi aprovado, seu coordenador tomou algumas providências.

- Atividade 1: o coordenador decidiu os membros que fariam parte da equipe envolvida na construção do modelo, comunicou prazos para a entrega final do trabalho e obteve o comprometimento da equipe em relação a este planejamento.

P2 - Análise de Fontes: por ser um conceito novo, foi necessário compreender conceitos e domínio do SPB. Para isto, foi usada a técnica T3 (Revisão da Literatura).

- Atividade 2: a primeira fonte identificada foi o próprio portal do SPB, uma vez que publicações sobre o domínio específico do modelo é escasso. O estudo permitiu uma compreensão inicial a respeito da estrutura do Portal, a identificação de papéis e práticas relacionadas às comunidades do SPB, e sobre terminologias utilizadas por seus membros. Análises foram conduzidas sobre estas fontes para ajudar construir o entendimento sobre o SPB.

- Atividade 4: outra fonte identificada foi uma edição especial da Revista InfoBrasil, publicada em maio de 2009, com matérias exclusivamente voltadas para a Qualidade para Desenvolvedores e Prestadores de Serviço no SPB.

- Atividade 7: o Portal SPB foi revisitado e a Revista InfoBrasil voltou a ser consultada de forma mais detalhada para aprofundar e consolidar $o$ entendimento a respeito de práticas o SPB. O resumo do resultado da execução desta Prática Sequencial pode ser visto na seção 3 deste artigo.

P3 - Estratégia de desenvolvimento: a definição da estratégia para o desenvolvimento do modelo SPB estabeleceu como a comunidade de interesse seria envolvida. 
- Atividade 3: foi definido que o modelo SPB seria desenvolvido de modo colaborativo, com a participação dos membros das comunidades do SPB e outros interessados.

- Atividade 5: foi determinado uma data e local para a realização de um workshop com a participação dos membros das comunidades do SPB e outros interessados para a apresentação e avaliação da versão preliminar do modelo SPB.

- Atividade 8: a estratégia para a construção do modelo SPB foi revisada pela equipe do subprojeto e outros meios de envolver a comunidade de interesse foram definidos. Assim, foi decidido que versões intermediárias do modelo seriam disponibilizadas para os membros das comunidades terem acesso e poderem colaborar e fornecer feedback. O meio definido para a interação entre a equipe e os membros das comunidades é via $5 \mathrm{CQualiBR}^{7}$. Esta é a comunidade do Portal SPB que compartilha com a sociedade os resultados obtidos com o Projeto Modelo de Referência do SPB [MCT 2007]. Ainda para viabilizar o feedback da comunidade de interesse, um fórum de discussão foi implementado na comunidade 5CQualiBR.

P4 - Projeto do Modelo: foram aplicadas as técnicas específicas T7 (Abstração de Processos) para apoiar na definição da estrutura do modelo e T9 (Brainstorming) com os membros da equipe para apoiar na definição do conteúdo da versão preliminar do modelo SPB.

- Atividade 6: foi decidido pelos membros da equipe que o modelo consolidado para ser usado como base para o formato do modelo SPB seria o CMMI-DEV. A partir do conhecimento adquirido na prática P2, foram sugeridas áreas de capacidade de processo para a versão preliminar do modelo SPB.

- Atividade 9: as áreas de capacidade sugeridas para a versão preliminar do modelo SPB foram priorizadas e duas foram selecionadas: (1) Gestão de Sugestões de Melhorias (backlog) e (2) Gestão de Comunidade. Atualmente, estas áreas de capacidade de processo foram renomeadas para Solicitação de Melhoria (SDM) e Gestão da Comunidade (GDC), respectivamente. Optou-se pelo formato do CMMI-DEV por ele ser um modelo reconhecido internacionalmente, facilitando a interpretação e uso do novo modelo.

P5 - Desenvolvimento da versão preliminar do Modelo: para a primeira versão do modelo SPB foi utilizada a técnica T7 (Abstração de Processos) para definir o conteúdo das duas áreas de capacidade de processo identificadas durante a execução da prática P4: Gestão da Comunidade (GDC) e Solicitação de Melhoria (SDM).

- Atividade 10: as áreas de capacidade de processo GDC e SDM foram descritas em formato alinhado à estrutura do modelo CMMI-DEV. Assim, cada uma é composta de um Propósito e um ou mais Objetivos. Cada Objetivo contém uma ou mais Práticas e ainda conta com exemplos de implementação de suas Práticas.

- Atividade 11: foi realizada a avaliação inicial da versão preliminar do modelo SPB com a aplicação da técnica T10 (Revisão pelos Pares) entre os membros da equipe do subprojeto.

\footnotetext{
${ }^{7}$ www.softwarepublico.gov.br/5cqualibr/
} 
P6 - Validação da versão preliminar do Modelo: a versão preliminar foi submetida a uma nova avaliação, desta vez realizada pelos membros das comunidades do SPB. A partir do feedback obtido, uma nova versão do modelo foi construída.

- Atividade 12: a versão preliminar do modelo foi apresentada durante o I Encontro Nacional do Software Público em um workshop realizado no dia 30 de outubro de 2009 em Brasília - DF. Esta ocasião proporcionou a interação da equipe do subprojeto com coordenadores de comunidade, desenvolvedores e com os usuários das soluções disponíveis no portal do SPB. Com o feedback obtido, a versão preliminar do modelo SPB foi revisada e publicada no portal SPB junto com um fórum específico interação entre membros das comunidades e equipe do subprojeto.

- Atividade 13: a partir da análise do relatório do workshop os membros da equipe do subprojeto puderam confirmar e elicitar outras novas áreas de capacidade de processo para serem incorporadas na próxima versão do modelo SPB. Assim, uma nova versão do modelo SPB foi construída e está em revisão.

Para a validação da versão preliminar do modelo SPB, está planejado sua aplicação no desenvolvimento de soluções do portal do SPB na forma de um estudo de caso. Os critérios desta validação ainda estão em elaboração. A técnica de estudo de caso foi selecionada a partir de como foram construídos os modelos estudados para construir o PRO2PI-MFMOD. Esta técnica mostrou-se também adequada para a realidade deste trabalho. Entretanto, o framework não é fechado, permitindo que ele seja estendido com outras técnicas para estudos experimentais.

P7 - Consolidação do Modelo: A partir dos resultados da atividade anterior, uma nova versão do modelo foi elaborada consolidando os resultados obtidos com o workshop em novas áreas de capacidade de processo. Esta versão é apresentada de forma reduzida na seção a seguir. O modelo será realmente consolidado com novos ciclos de atividades, que envolverão uma maior divulgação, utilização efetiva e melhoria continua do modelo.

- Atividade 14: a versão 3.0 do modelo foi avaliada e consolida os resultados do workshop em duas áreas de capacidade de processo revisadas somadas a sete novas áreas.

\section{O Modelo de Capacidade de Processo para o SPB}

Esta seção apresenta a estrutura e um resumo do conteúdo da versão 3.0 do modelo de capacidade de processo para o SPB.

\subsection{Estrutura do Modelo de Capacidade de Processo para o SPB}

As áreas de capacidade de processo do modelo SPB descrevem um conjunto de práticas para orientar as comunidades do portal SPB a melhorar a qualidade das soluções disponibilizadas. As comunidades do SPB tem a liberdade de definir a forma de "como" implementar cada prática do modelo. As áreas de capacidade de processo estão descritas neste trabalho com os seguintes componentes:

- Propósito: Descreve a finalidade da área de capacidade de processo;

- Objetivo: É o alvo que se quer atingir por meio da aplicação das práticas; 
- Prática: Orienta na maneira de realizar as atividades, não se preocupando com o "como" fazer, mas com "o que" fazer;

O modelo SPB ainda conta com o componente "exemplos" que apresenta sugestões de "como" uma prática pode ser implementada, porém ele não foi detalhado neste artigo. A arquitetura do modelo SPB foi definida com base na estrutura do CMMIDEV. Ela pode vir a ser refinada caso durante a execução dos futuros estudos de caso seja observada esta necessidade como uma forma do modelo estar mais alinhado às características das comunidades de interesse.

\subsection{Conteúdo do Modelo de Capacidade de Processo para o SPB}

A versão 3.0 do modelo SPB descreve nove áreas de capacidade de processo:

1. Gestão Estratégica (GES): busca ajudar as comunidades a atingir os objetivos do SPB.

Objetivo GES 1 - Alinhamento dos Objetivos da Comunidade com Objetivos do SPB:

$>$ GES 1.1: Conhecer os objetivos do SPB;

$>$ GES 1.2: Estabelecer e manter uma estratégia de alinhamento dos objetivos da comunidade com os objetivos do SPB;

$>$ GES 1.3: Definir e executar ações para induzir a Comunidade ao atendimento dos objetivos do SPB;

> GES 1.4: Monitorar o alinhamento dos objetivos da comunidade com os objetivos do Software Público Brasileiro;

$>$ GES 1.5: Definir e acompanhar ações corretivas para os objetivos da comunidade.

2. Gestão da Comunidade (GDC): visa auxiliar o responsável por gerenciar a comunidade nas definições e acompanhamento das atividades da comunidade.

Objetivo GDC 1 - Gestão da Comunidade Preparada:

$>$ GDC 1.1: Estabelecer e manter os objetivos da comunidade;

$>$ GDC 1.2: Estabelecer e manter uma estratégia para atingir os objetivos da Comunidade;

$>$ GDC 1.3: Estabelecer e manter regras e modo de operação da Comunidade alinhada a sua estratégia;

$>$ GDC 1.4: Estabelecer e manter um mecanismo para apoiar o funcionamento da Comunidade.

Objetivo GDC 2 - Atividades de Gestão da Comunidade Realizadas e Acompanhadas:

GDC 2.1: Realizar as atividades de Gestão da Comunidade;

$>$ GDC 2.2: Monitorar o dia a dia da Comunidade em relação à estratégia;

$>$ GDC 2.3: Monitorar a estratégia em relação aos objetivos;

$>$ GDC 2.4: Definir e acompanhar ações corretivas para alinhar a estratégia da comunidade com seus próprios objetivos. 
3. Gestão do Conhecimento (GCH): vem ajudar as comunidades a assegurar que o conhecimento individual, informações e os produtos gerados pela comunidade sejam gerenciados e compartilhados.

Objetivo GCH 1 - Itens de Conhecimento da Comunidade Gerenciados:

> GCH 1.1: Estabelecer e manter um mecanismo de gestão do conhecimento;

$>$ GCH 1.2: Estabelecer e manter uma estratégia de gestão do conhecimento;

$>$ GCH 1.3: Capturar os itens de conhecimento;

$>$ GCH 1.4: Disseminar o conhecimento;

$>$ GCH 1.5: Melhorar os itens de conhecimento.

4. Gestão de Reutilização (GDR): visa ajudar as comunidades a viabilizar a reutilização de produtos de trabalho.

Objetivo GDR 1 - Produtos de Trabalho Reutilizáveis são Gerenciados:

> GDR 1.1: Definir uma estratégia de reutilização de produtos de trabalho;

$>$ GDR 1.2: Estabelecer um mecanismo para apoiar a gestão de reutilização;

$>$ GDR 1.3: Implementar o programa de reutilização;

D GDR 1.4: Registrar dados de uso dos produtos de trabalho reutilizáveis;

> GDR 1.5: Notificar usuários dos produtos de trabalho reutilizáveis sobre atualizações.

5. Gestão de Relacionamento com Clientes (GRC): Clientes de uma comunidade do SPB podem ser os usuários, os solicitantes de melhorias, prestadores de serviço ou desenvolvedores de software. Esta área busca ajudar as comunidades a gerenciar o relacionamento com seus clientes

Objetivo GRC 1 - Relacionamento com Clientes Gerenciado:

$>$ GRC 1.1: Conhecer os Clientes;

$>$ GRC 1.2: Obter feedback dos clientes;

$>$ GRC 1.3: Identificar os objetivos de comunicação com os clientes;

$>$ GRC 1.4: Definir uma estratégia de comunicação com os clientes;

$>$ GRC 1.5: Estabelecer e manter a comunicação com os clientes.

6. Solicitação de Melhoria (SDM): tem a finalidade de auxiliar os responsáveis pelas comunidades no gerenciamento das sugestões de melhorias para as soluções.

Objetivo SDM 1 - Solicitações de Melhoria Gerenciadas:

$>$ SDM 1.1: Definir a estratégia de gerenciar as sugestões de melhorias;

$>$ SDM 1.2: Estabelecer e manter um mecanismo para gerenciar a solicitação de melhoria;

SDM 1.3: Receber, identificar e registrar solicitação de melhoria;

$>$ SDM 1.4: Aceitar a solicitação de melhoria;

$>$ SDM 1.5: Monitorar a lista de solicitação de melhoria.

7. Fornecimento de Melhoria (FDM): tem a finalidade de auxiliar os responsáveis pelas comunidades a gerenciar de forma colaborativa e sustentável o fornecimento de melhorias para a solução. 
Objetivo FDM 1 - Melhorias são Fornecidas para a Solução:

> FDM 1.1: Orientar a seleção da solicitação de melhoria para o release;

> FDM 1.2: Registrar responsável por implementar a solicitação de melhoria;

> FDM 1.3: Acompanhar a solicitação de melhoria até o final de sua implementação.

8. Integração e Liberação da Solução (ILS): busca auxiliar os membros e responsáveis das comunidades a montar a solução a partir de componentes da solução, assegurar que a melhoria integrada à solução execute as funcionalidades solicitadas de forma apropriada e liberar a solução deixando-a disponível para uso pelos demais membros da comunidade.

Objetivo ILS1 - Integração da Solução Preparada:

> ILS 1.1: Determinar o procedimento e a sequência da integração;

$>$ ILS 1.2: Estabelecer mecanismo de integração da solução;

> ILS 1.3: Estabelecer procedimentos para a integração da solução.

Objetivo ILS2 - Compatibilidade das Interfaces Assegurada:

> ILS 2.1: Revisar Descrições de Interfaces para Assegurar Completude;

$>$ ILS 2.2: Gerenciar Interfaces.

Objetivo ILS3 - Componentes da Solução Montados e Solução Liberada:

> ILS 3.1: Confirmar se os componentes da solução estão prontos para serem integrados;

> ILS 3.2: Montar componentes da solução;

> ILS 3.3: Avaliar componentes da solução montados e registrar os resultados;

> ILS 3.4: Empacotar e liberar a solução ou o componente da solução.

9. Treinamento na Tecnologia e na Solução (TTS): buscando capacitar os membros das comunidades e ampliar a rede de manutenção da solução auxiliando o responsável por gerenciar a comunidade na capacitação de seus membros nas habilidades e no conhecimento necessário para o uso da solução e das tecnologias adotadas para mantê-la.

Objetivo TTS 1 - Infraestrutura de Treinamento para a Comunidade Estabelecida e Mantida:

$>$ TTS 1.1: Definir uma estratégia para o treinamento na solução e nas tecnologias adotadas;

> TTS 1.2: Identificar as necessidades de treinamento na solução e nas tecnologias adotadas;

$>$ TTS 1.3: Estabelecer infraestrutura para treinamento na solução e nas tecnologias adotadas.

Objetivo TTS 2 - Treinamento Necessário Disponibilizado:

$>$ TTS 2.1: Disponibilizar a infraestrutura para treinamento na solução e nas tecnologias adotadas;

$>$ TTS 2.2: Manter registros de treinamento dos membros da comunidade na solução e nas tecnologias adotadas. 
Esta seção apresentou a versão 3.0 do modelo de capacidade de processo para o SPB. Em breve, esta versão do modelo poderá ser vista na íntegra, incluindo os exemplos de implementação de suas práticas, na comunidade 5CQualiBR.

\section{Conclusão}

Este trabalho apresentou a aplicação de um método definido a partir do PRO2PIMFMOD [Salviano et al. 2009b] para a construção do modelo de capacidade de processo para o SPB. Uma avaliação do método e as técnicas utilizadas para apoiar a execução do método também foram discutidas. A versão preliminar do modelo construído foi apresentada, bem como os resultados de sua primeira avaliação por meio de um workshop com a comunidade de interesse.

A versão atual deste modelo foi elaborada com base nas observações obtidas por meio do workshop, pelos feedbacks coletados a partir do fórum de discussão e reuniões entre os membros da equipe do subprojeto. Estudos de caso de aplicação da nova versão do modelo estão planejados para avalia-lo em projetos pilotos e assim identificar seus pontos fortes, pontos fracos e oportunidades de melhoria. A partir destes resultados, novas versões do modelo para o SPB serão desenvolvidas.

\section{Agradecimentos}

Os autores agradecem ao FINEP - Financiadora de Estudos e Projetos, por financiar e apoiar este trabalho sob o projeto 0489/08: Modelo de Referência para o Software Público Brasileiro.

\section{Referências}

Alves, A. M., Stefanuto, G. N., Castro, P. F. D. e Varani, S. A. (2009) "Software Público Brasileiro: muito além do compartilhamento de software”, In: InfoBrasil ano II - No 7.

Anacleto, A., Gresse von Wangenheim, C., Salviano, C. F., Savi, R. (2004) "A Method for Process Assessment in Small Software Companies", In: Proceedings of the Fourth International SPICE Conference, p. 69-76.

AUTOMOTIVE SIG (2008) “Automotive SPICE Process Assessment Model”, Final Release, v4.4, 46 pages, (C) The SPICE User Group, 01 August 2008 (disponível a partir de http://www.automotivespice.com, last accessed 23/05/2009).

Bunge, M. (1974) "La ciencia, su método y su filosofia” Buenos Aires: Siglo Veinte.

Fuggetta A. (2000) "Software Process: A Roadmap, in Proceedings of Future of Software Engineering Workshop", In: ICSE, Limerick, Ireland, pp. 25-34.

Freitas, C. (2009) "Pesquisa avalia impacto do uso do Portal do SPB", In: InfoBrasil ano II - $\mathrm{N}^{\mathrm{o}} 7$.

Hauck, J. C. R., Gresse von Wangenheim, C., Wangenheim, A. Von. (2008) "Development of a Process Reference Model for Telemedicine Software Development", In:Proceedings of the EuroSPI 2008 Doctoral Symposium. Berlin : Logos Verlag Berlin GmbH, . p. 39-48.

Humphrey, W. S. (1995) "Introducing the personal software process". Springer Netherlands: Annals of Sofware Engineering, v. 1, n. 1, p. 311-325. 
ISO/IEC (2003), "Information Technology - Software Process Assessment", Parts 1-9, The International Organization for Standardization and the International Electrotechnical Commission, v. ISO/IEC 15504.

ITGI IT Governance Institute. (2005) “COBIT”, 4th Edition, http://www.isaca.org

Júnior, J. L. C., Rodrigues, M. A., Alves, A. M. (2009) “Um Modelo de Referência para o Software Público Brasileiro, In: InfoBrasil ano II - No 7.

MCT (2007) "Projeto Modelo de Referência do Software Público Brasileiro - SPB" Ministério da Ciência e Tecnologia - Proposta - Versão 2.3, p. 1-58.

Meffe, C. (2009) "Portal do Software Público comemora 2 anos e integra 40 mil usuários”, In: InfoBrasil ano II - No 7.

Paulk, M. C., Weber, C.W., Curtis, B., Chrissis, M. B. (1994) "The Capability Maturity Model - Guidelines for Improving the Software Process", Addison-Wesley, 441 pages.

Pickler, K. K.; Gresse von Wangenheim, C., Salviano, C. F. (2005) "MARES-MINI/EI: Propondo um Método de Avaliação de Processo de Software em Micro Empresas Incubadas", In: IV Simpósio Brasileiro de Qualidade de Software, Porto Alegre.

Salviano, C.F. (2006) "Uma proposta orientada a perfis de capacidade de processo para evolução da melhoria de processo de software". Tese de doutorado pela UNICAMP, Faculdade de Engenharia Elétrica e de Computação.

Salviano, C. F., Zoucas, A. C. e Zapelini, C. Z. (2009a) "Qualidade para Desenvolvedores e Prestadores de Serviço no Software Público Brasileiro”, In: InfoBrasil ano II - $\mathrm{N}^{\mathrm{o}} 7$.

Salviano, C. F., Zoucas, A. C., Silva, J. V. L., Alves, A. M., Wangenheim, C. G. e Thiry, M. (2009b) "A Method Framework for Engineering Process Capability Models", In: EuroSPI, The 16th European Systems and Software Process Improvement and Innovation, Industry Proc., pp. 6.25-6.36, 2-4, Spain.

SEI (2006) "CMMI for Development: Version 1.2: CMMI-DEV", Software Engineering Institute USA.

Silva, J. V. L., Nabuco, O. F., Salviano, C. F., Reis, M. C., Maciel F. R. (2007) "Strategic Management in University Research Laboratories Towards a Framework for Assessment and Improvement of R\&D Management", In: SPICE Conf. Proc., South Korea.

SOFTEX (2009) "MR-MPS - Melhoria de Processo do Software Brasileiro: Guia Geral," Associação para Promoção da Excelência do Software Brasileiro, SP.

Tsukumo, A. N., Santos, R. V. M., Marinho ,W., Silva, L. P., Salviano, C. F. (2006) "Uma Estratégia para Melhoria de Processo de Desenvolvimento de Software Baseado em Componentes", In: V SBQS, Vila Velha - ES, Brasil.

Zoucas, A. C., Thiry, M., Salviano, C. F. (2009) “Técnicas para Engenharia de Modelos de Capacidade de Processo de Software", In: IWASE, Proc. of the 2nd International Workshop on Advanced Software Engineering. Santiago, Chile. p. 11-18. 